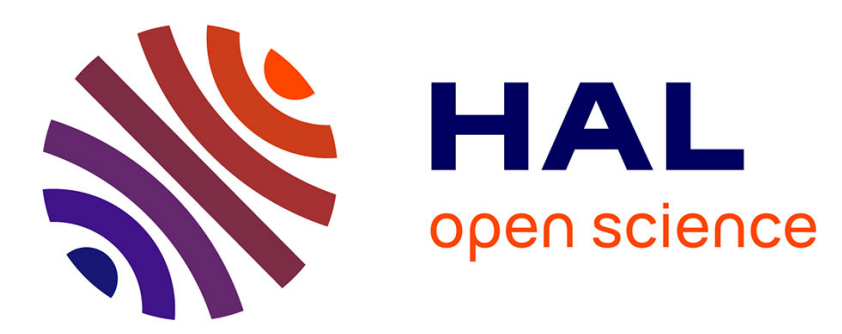

\title{
Improvement of the off-diagonal magnetoimpedance sensor white noise
}

Michal Malatek, Basile Dufay, Sébastien Saez, Christophe Dolabdjian

\section{To cite this version:}

Michal Malatek, Basile Dufay, Sébastien Saez, Christophe Dolabdjian. Improvement of the off-diagonal magnetoimpedance sensor white noise. Sensors and Actuators A: Physical , 2013, 204, pp.20-24. 10.1016/j.sna.2013.10.001. hal-00877644

\section{HAL Id: hal-00877644 https://hal.science/hal-00877644}

Submitted on 29 Oct 2013

HAL is a multi-disciplinary open access archive for the deposit and dissemination of scientific research documents, whether they are published or not. The documents may come from teaching and research institutions in France or abroad, or from public or private research centers.
L'archive ouverte pluridisciplinaire HAL, est destinée au dépôt et à la diffusion de documents scientifiques de niveau recherche, publiés ou non, émanant des établissements d'enseignement et de recherche français ou étrangers, des laboratoires publics ou privés. 


\title{
Improvement of the off-diagonal magnetoimpedance sensor white noise
}

\author{
M. Malatek ${ }^{\mathrm{a}, \mathrm{b}, \mathrm{c}}$, B. Dufay ${ }^{\mathrm{a}, \mathrm{b}, \mathrm{c}, \mathrm{d}, *}, \mathrm{~S} . \mathrm{Saez}^{\mathrm{a}, \mathrm{b}, \mathrm{c}}$, C. Dolabdjian ${ }^{\mathrm{a}, \mathrm{b}, \mathrm{c}}$ \\ ${ }^{a}$ Normandie Univ, France \\ ${ }^{b} U C B N$, GREYC, F-14032 Caen, France \\ ${ }^{c}$ CNRS, UMR 6072, F-14032 Caen, France \\ ${ }^{d}$ École Polytechnique de Montréal, département de génie physique $\mathscr{G}$ regroupement québécois des matériaux de pointe, \\ Montréal, Québec, Canada H3C3A7
}

\begin{abstract}
This work deals with the analysis of noise sources of the off-diagonal magneto-impedance sensor with stressannealed amorphous ribbon. This experimental analysis shows that the equivalent magnetic noise spectral density is mostly due to the electronic conditioning circuitry that operates the sensor. The electronic noise was then reduced with employment of an improved excitation-detection technique for our sensor and an equivalent magnetic noise spectral density below $1 \mathrm{pT} / \sqrt{\mathrm{Hz}}$ in white noise region has been reached. Indeed, the equivalent magnetic white noise level of the output signal decreased from $5 \mathrm{pT} / \sqrt{\mathrm{Hz}}$ to $560 \mathrm{fT} / \sqrt{\mathrm{Hz}}$. Nevertheless, this noise level is still limited by the electronic conditioning, leaving room for further improvement.
\end{abstract}

Keywords: Off-diagonal magnetoimpedance, amorphous ribbon, magnetic field sensor, noise.

\section{Introduction}

The giant magnetoimpedance (GMI) effect is a phenomena observed in ferromagnetic conductors driven by high frequency current and exposed to external magnetic field. Basically, the ac permeability, and so the impedance, of such material changes when exposed to an external magnetic field variation [1]. In the last few decades it has attracted considerable attention due to its strong potential in high sensitivity magnetometry.

Diagonal (or classical) GMI effect is observed as an impedance change of the magnetic materials related to an external magnetic field variation. Another approach to improving sensitivity is the choice of a two port network configuration, in which the GMI magnetic material is associated with a pick-up coil $[2,3]$. In such a case, a change of the apparent (off-diagonal) impedance, $Z_{12}=V_{2} / I_{1}$ (see Fig. 1), occurs versus an external magnetic field variation. Sensitivity of the off-diagonal GMI device to the external magnetic field can be magnified with a large number of turns of the pick-up

\footnotetext{
* Corresponding author

Email address: basile.dufay@unicaen.fr (B. Dufay)
}

coil. It was also proven that parameters like linearity and temperature stability are also improved [4] compared to the classical (diagonal) GMI devices. Different names, like Inverse Wiedemann effect [5] or Orthogonal fluxgate sensor in fundamental frequency $[2,6]$, were sometimes used.

In this paper, following up on the work from ref. [7], main attention is paid to the analysis of noise sources and to the improvement of the sensor white noise level.Regarding the output noise spectral density, it usually can be separated in a low frequency excess noise, named $1 / f$ noise, and a white noise floor $[8,9]$. The white noise is mostly expected as a limit given by the electronic noise while the intrinsic noise of the sensing element is expected to be more of the $1 / f$ behavior. However, exact differentiation of noise sources and their spectrum is a difficult task.

Investigation of GMI sensors noise performance is not a novelty. Several researches reported on noise level achieved with GMI devices [10]. However, a conceptual improvement of noise properties of GMI sensing element was first given by MeLo et al. [11]. Later, a detailed description and a model of noise contributors of classical GMI sensors were presented by DiNG et al. [9]. In the last 


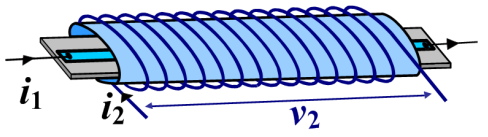

(a)

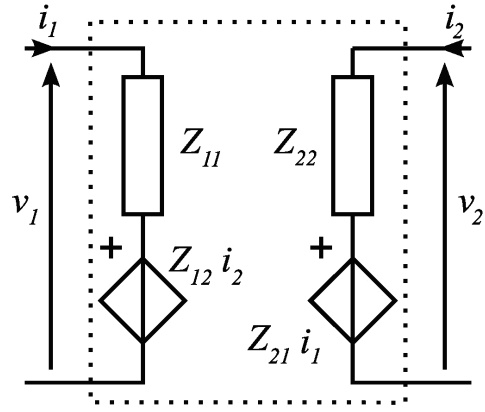

(b)

Figure 1: (a) Sensing element (an amorphous ribbon with associated pickup coil). $i_{1}$ and $v_{2}$ are the AC excitation current and the induced pick-up voltage, respectively. (b) Associated electrical two port network model.

mentioned work, experimental results on soft amorphous wires $(2 \mathrm{~cm}$ long and $30 \mu \mathrm{m}$ in diameter) were also presented and a white noise level of $3 \mathrm{pT} / \sqrt{\mathrm{Hz}}$ at $1 \mathrm{kHz}$ was achieved. A low frequency noise, around $100 \mathrm{pT} / \sqrt{\mathrm{Hz}}$, was obtained at $1 \mathrm{~Hz}$. The same noise analysis was carried out for off-diagonal GMI sensors [12] leading to measured noise performances slightly lower than $1 \mathrm{pT} / \sqrt{\mathrm{Hz}}$ in white noise region and $35 \mathrm{pT} / \sqrt{\mathrm{Hz}}$ at $1 \mathrm{~Hz}[13](2.5 \mathrm{~cm}$ long and $3 \mathrm{~mm}$ in diameter). Previous results dedicated to the case of off-diagonal GMI sensor using ribbon with creep-induced anisotropy $(11 \mathrm{~cm}$ long, $1.2 \mathrm{~mm}$ wide and $17 \mu \mathrm{m}$ thick), a white noise level of $8 \mathrm{pT} / \sqrt{\mathrm{Hz}}$ and $17 \mathrm{pT} / \sqrt{\mathrm{Hz}}$ at $1 \mathrm{~Hz}$ were reached [7]. More recently, BUTTA et al. have presented a white noise level of $2.5 \mathrm{pT} / \sqrt{\mathrm{Hz}}$ for an off-diagonal GMI sensor based on a $45 \mathrm{~mm}$ long and $120 \mathrm{\mu m}$ Co-rich magnetic wire [14].

The paper is organized as follows. Section 2 describes the sensor operation, the experimental setup and its noise model, based on previous development [8]. The electronic conditioning for low-noise measurement performance is also pointed out there. Then, results of an improved set-up are given in section 3 , which is followed by a conclusion.

\section{Classical set-up}

This section deals with the characterization of the sensitivity and noise performances of the measuring chain in a classical set-up illustrated on Fig. 2. The sensor is made of $11 \mathrm{~cm}$ long, $1.2 \mathrm{~mm}$ wide and $17 \mu \mathrm{m}$ thick ribbon of $\mathrm{Co}_{67} \mathrm{Fe}_{4} \mathrm{Si}_{8} \mathrm{~B}_{14} \mathrm{Cr}_{7}$ produced by planar flow casting. The ribbon, annealed under tensile stress of $50 \mathrm{MPa}$ for 10 minutes at $390{ }^{\circ} \mathrm{C}$, is placed on non-magnetic plate and is electrically connected to a coaxial cable. A pick-up coil of 290 turns is wound on fiberglass tube of $9 \mathrm{~mm}$ in diameter, in which the ribbon is inserted.

Taking into account all electronic noise sources appearing in the measuring chain, and considering that the sensing device is fully described by means of classical two port network parameter [12] and its associated impedance matrix (Fig. 1b), the expected equivalent magnetic white noise spectral density of the sensor, at the magnetic field working point, $B_{0}$, is given by $[8,9]$

$$
\begin{aligned}
& \left.b_{n}^{2}(f)\right|_{B=B_{0}} \approx 2\left(\frac{R_{1}+\left|Z_{11}\left(f_{0}\right)\right|}{e_{g 1} \frac{\partial\left|Z_{21}\left(f_{0}\right)\right|}{\partial B}}\right)^{2}\{ \\
& \quad\left(\frac{\left|Z_{21}\left(f_{0}\right)\right|}{R_{1}+\left|Z_{11}\left(f_{0}\right)\right|}\right)^{2}\left[\left(\frac{e_{g 1}}{D_{y}}\right)^{2}+e_{n R 1}^{2}\left(f_{0}\right)\right] \\
& \left.\quad+\left|Z_{22}\left(f_{0}\right)\right|^{2} i_{n-L I A}^{2}\left(f_{0}\right)+e_{n-L I A}^{2}\left(f_{0}\right)\right\}
\end{aligned}
$$

where $R_{1}$ is the generator output impedance, $Z_{i j}$ are the impedance matrix parameters of the sensing element at the static working point $B_{0}, e_{g 1}$ is the amplitude of the excitation generator, $i_{n-L I A}$ and $e_{n-L I A}$ are the current and voltage noise of the Lock-In Amplifier (LIA), and $D_{y}$, is the generator dynamic range. This equation shows how each element contributes to the equivalent magnetic noise level of the sensor. Notice that noises coming from the lead battery power fluctuations and the intrinsic magnetic sensor noise are neglected here.

All noise measurements were accomplished with sensor placed into the GREYC magnetic-shielded room excluding all noise contribution from the surrounding environment. The AC current signal, $i_{1}$, 


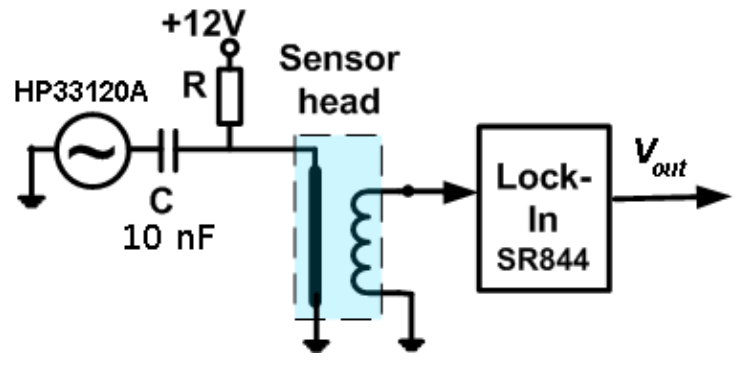

Figure 2: Sensing head and associated conditioning electronic set-ups. $R$ is a DC bias resistance. The lock-in amplifier is synchronized to the excitation frequency $f_{0}$.

was driven by a waveform generator HP33120A at frequency, $f_{0}\left(f_{0} \approx 500 \mathrm{~Hz}\right)$. The DC bias current came from $12 \mathrm{~V}$ lead battery. In addition, the sensor was exposed to an external low frequency sine wave magnetic field, $h_{e x t}$, induced by Helmholtz coils. This field, of nT-range magnitude at frequency, $f_{1}$, around $10 \mathrm{~Hz}$, was used as reference signal for sensitivity calibration. The output signal, $v_{2}$, of the off-diagonal GMI sensing element is therefore a classical AM modulated signal with high frequency carrier at $f_{0}$ and modulating sine wave signal at frequency $f_{1}$. The modulating signal is extracted thanks to a synchronous demodulation accomplished with high-frequency $S R$-844 lock-in amplifier (Fig. 2). Output noise spectral density of the measuring chain was recorded by a low frequency spectral analyzer HP3562A.

Firstly, the effect of the AC amplitude current excitation was investigated. In this experiment, the AC current amplitude, $I_{1}$, was increased up to $50 \mathrm{~mA}_{\mathrm{RMS}}$ (sinewave) and was then set back to a value of $10 \mathrm{~mA} A_{\text {RMS }}$. This sequence is shown in Fig. 3, where correlation between sensitivity and AC amplitude is depicted.

As it can be seen, for three different DC bias current values, the sensitivity increases linearly with the amplitude of the $\mathrm{AC}$ excitation up to a certain point. At the largest excitation of $50 \mathrm{~mA}_{\mathrm{RMS}}$, the sensitivity was not measurable for DC bias current of $1 \mathrm{~mA}$ (solid squares) and dropped down by $25 \%$ for DC bias current of $5 \mathrm{~mA}$ (solid circles). Afterward, when the amplitude of excitation current was set back to $10 \mathrm{~mA}_{\mathrm{RMS}}$, the sensitivity of the sensor was lower than the original one for these two DC bias currents (empty marks at $10 \mathrm{~mA}$ value on $\mathrm{x}$-axis). Nevertheless, it appears that the sensor re-

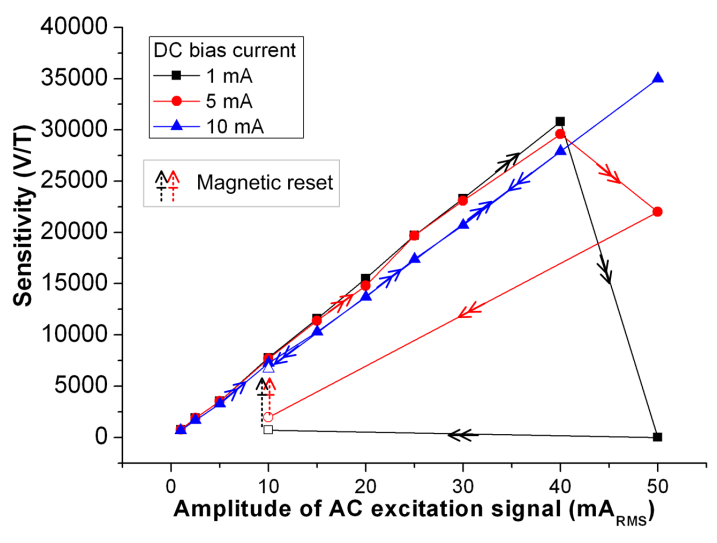

Figure 3: Sensitivity versus excitation current amplitude for three different values of the DC bias current. The excitation frequency was set to $490 \mathrm{kHz}$. Arrows indicate the direction of AC amplitude development within the experiment. The magnetic reset consists of a strong external applied magnetic field pulse.

trieves its original behavior after being exposed to a strong external magnetic field pulse (labeled as magnetic reset in Fig. 3). In the case of DC bias current of $10 \mathrm{~mA}$, the sensor works properly even at $50 \mathrm{~mA}_{\mathrm{RMS}}$ and keeps linearity between sensitivity and excitation current amplitude. The sensitivities at $10 \mathrm{~mA}_{\mathrm{RMS}}$ excitation, before and after the highamplitude excitation, are nearly the same in that case. In all the following we make sure to avoid anomalies occuring at high excitation current.

The output noise spectral densities for the different amplitudes of AC excitation are depicted on Fig. 4a.

These experiments highlight the weakness of the measurement set-up, which is the dynamic reserve of the lock-in amplifier. Each record of the Fig. 4a was acquired with lock-in amplifier gain set to the most suitable value (i.e. a maximal gain with nonsaturated/non-overloaded input filters). Thus, gain and dynamic range of the amplifier were not the same for the different excitation parameters of the sensor. Looking at the output voltage noise spectral densities (Fig. 4a), one can see that the noise floor reaches value around $30 \mathrm{nV} / \sqrt{\mathrm{Hz}}$ for lock-in amplifier ranges of $3 \mathrm{mV}$ and $10 \mathrm{mV}$, whereas it jumps up to about $100 \mathrm{nV} / \sqrt{\mathrm{Hz}}$ when the measurement range is $30 \mathrm{mV}, 100 \mathrm{mV}, 300 \mathrm{mV}$ or $1 \mathrm{~V}$. Obviously, the white noise level in our experiments is determined by the lock-in amplifier dynamic range and 


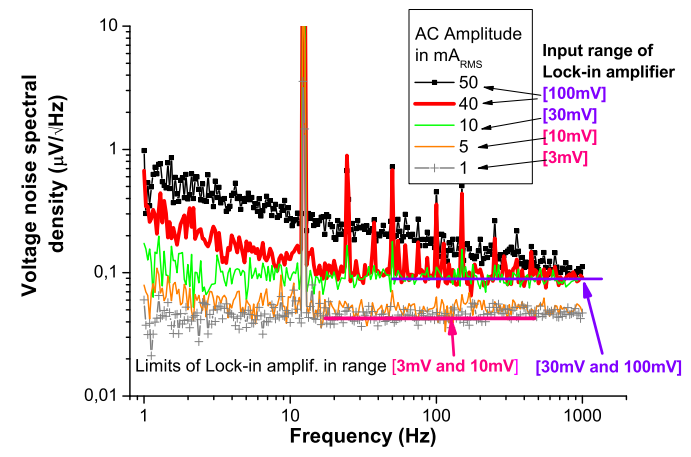

(a)

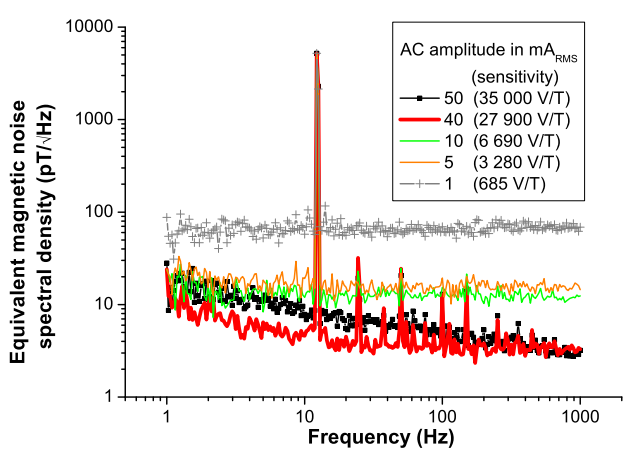

(b)

Figure 4: (a) Output voltage noise spectral densities $(\mu \mathrm{V} / \sqrt{\mathrm{Hz}})$ of the basic measurement set-up for a $10 \mathrm{~mA}$ DC bias current and different amplitudes of sine wave excitation current at $f_{0}=490 \mathrm{kHz}$. Input range of the lock-in amplifier is written in square brackets. (b) Equivalent magnetic noise spectral densities of the sensor $(\mathrm{pT} / \sqrt{\mathrm{Hz}})$ after dividing by the sensitivity.

hides the other electronic noise sources as well as the intrinsic noise of the sensor. Figure $4 \mathrm{~b}$ shows the equivalent magnetic noise spectral density expressed in units of $\mathrm{T} / \sqrt{\mathrm{Hz}}$ after divided by the sensitivity. To summarize, we observe, for used ribbon sensor in the classical electronic set-up, the following points

- best noise performance was achieved for $\mathrm{AC}$ current excitation amplitudes around $40 \mathrm{~mA}_{\mathrm{RMS}}$,

- the measured white noise level of $4 \mathrm{pT} / \sqrt{\mathrm{Hz}}$ is limited by the lock-in amplifier noise and its dynamic reserve,

- a further increase of the sensitivity must not necessarily reduce the equivalent magnetic noise spectral density of the measuring chain since at excitation current of $50 \mathrm{~mA}_{\mathrm{RMS}}$ the sensitivity rises proportionally (Fig. 3), but the noise background grows even more (Fig. 4b).

Similar behaviors were also recently reported in [14].

\section{Improved set-up}

Previous section highlights the problematic part of the signal conditioning chain due to the dynamic of the demodulation stage. In order to operate at a lower measurement range, with lower white

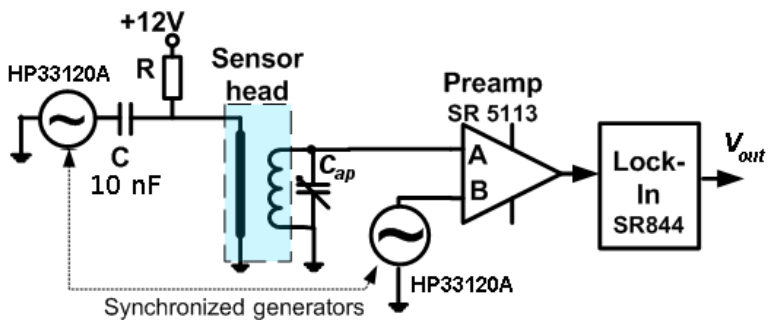

Figure 5: (a) Sensor head operation in the improved signal conditioning set-up. The resistor $R$ allows to set a DC bias current flowing through the ribbon. The additional generator on input $\mathrm{B}$ of the preamplifier is used in order to suppress the carrier of the AM signal from the sensing element. The lock-in amplifier is synchronized to the excitation frequency, $f_{0}$. The capacitor, $C_{a p}$, in parallel to the pick-up coil, tunes the sensor at the resonance, according to the excitation frequency. 


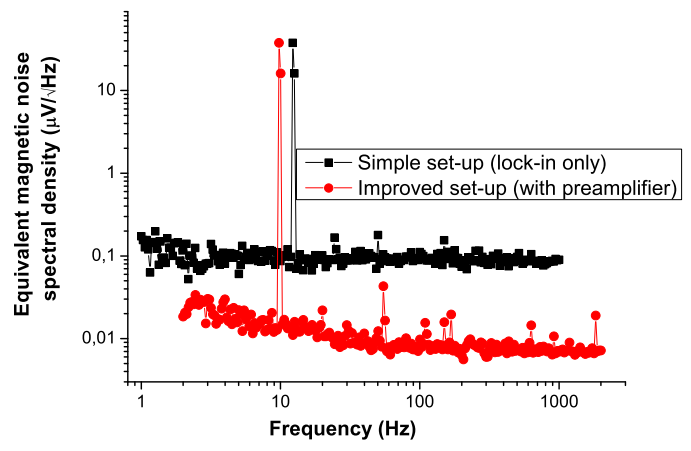

Figure 6: Output voltage noise spectral density for the two used set-up considering an excitation current amplitude of $10 \mathrm{~mA}_{\mathrm{RMS}}$ and a static bias current of $10 \mathrm{~mA}$ in both cases.

noise level, of the lock-in amplifier, an improved set-up was realized by means of differential low noise preamplifier and additional generator. The schematic of this set-up is shown on Fig. 5. The phase and amplitude of the second generator is adjusted in order to be subtracted from the pick-up voltage, $v_{2}$, of the sensor. By this way, an AM signal with suppressed carrier is obtained at the output of the preamplifier allowing to reduce the lock-in required dynamic range, still having the same amplitude of modulated signal. Improvement of the white noise level is shown on Fig. 6, where both records (in the original and the improved configurations) are compared. Here, the sensor is driven by $10 \mathrm{~mA}_{\mathrm{RMS}}+10 \mathrm{~mA}_{\mathrm{DC}}$ current.

The capacitor, $C_{a p}$, in parallel to the pick-up coil, tunes the sensor into the resonance according to the excitation frequency, which increases the sensor sensitivity and so the SNR [7]. Similarly as Eq. (1), the expected equivalent magnetic white noise level of the sensor, at the magnetic field working operation, $B_{0}$, is given by

$$
\begin{aligned}
& \left.b_{n}^{2}(f)\right|_{B=B_{0}} \approx 2\left(\frac{R_{1}+\left|Z_{11}\left(f_{0}\right)\right|}{E_{g 1} \frac{\partial\left|Z_{21}\left(f_{0}\right)\right|}{\partial B}}\right)^{2}\{ \\
& \left(\frac{\left|Z_{21}\left(f_{0}\right)\right|}{R_{1}+\left|Z_{11}\left(f_{0}\right)\right|}\right)^{2}\left[2\left(\frac{E_{g 1}}{D_{y}}\right)^{2}+e_{n R_{1}}^{2}\left(f_{0}\right)\right] \\
& +e_{n R_{2}}+\left|Z_{22}\left(f_{0}\right)\right|^{2} i_{n-b u f f}^{2}\left(f_{0}\right) \\
& \left.+e_{n-b u f f}^{2}\left(f_{0}\right)+\frac{e_{n-L I A}^{2}}{G^{2}}\right\}
\end{aligned}
$$

where $G, i_{n-b u f f}$ and $e_{n-b u f f}$ are the gain, the current noise source and voltage noise source of

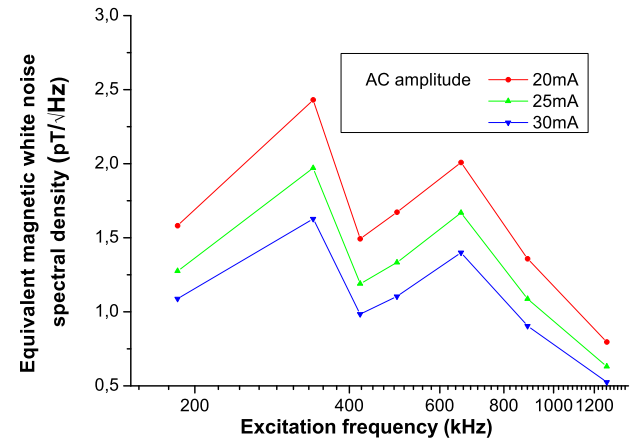

Figure 7: Equivalent magnetic white noise levels versus excitation current frequency, for several excitation current amplitudes (measured with the improved measurement set-up described on Fig. 5). Results are obtained with the new pick-up coil, which increase the filling factor, tuned with a parallel capacitor.

the preamplifier, respectively. Several noise measurements were accomplished at various excitation frequencies to find the best regime of operation of the sensor. Experimentally, the best noise performances, with a white noise level of $0.7 \mathrm{pT} / \sqrt{\mathrm{Hz}}$, were achieved at $f_{0}=360 \mathrm{kHz}$, for an excitation current amplitude of $30 \mathrm{~mA}_{\mathrm{RMS}}$ and a $10 \mathrm{~mA}_{\mathrm{DC}}$ bias current.

Following these results, a new pick-up coil, with same number of turns as the previous one and smaller diameter $(6.7 \mathrm{~mm})$, was used in order to compare the effect of a higher filling factor. Fig. 7 shows the measured equivalent magnetic white noise levels, in units of $\mathrm{pT} / \sqrt{\mathrm{Hz}}$, versus the frequency of excitation. Capacitance, $C_{a p}$, was tuned accordingly to each excitation frequency. Employing the new pick-up coil, we achieve minimum white noise level of around $0.6 \mathrm{pT} / \sqrt{\mathrm{Hz}}$ at an excitation frequency of $1.27 \mathrm{MHz}$.

The output voltage noise spectral density of the sensor operated under these optimal conditions is given on Fig. 8a (gray empty triangles), which is also compared to the noise spectral density from generator (red line). The system noise was evaluated by means of additional generator (replacing the sensor). The amplitude and phase of the generator output were adjusted in the way to match with the signal in the second input of the preamplifier. It clearly appears that the generator noise mostly contributes to the total noise level of the measur- 
ing chain. The black minus marks in Fig. 8 show the noise floor of the detection stage, which was acquired when the amplifier's input was terminated with $50 \Omega$ impedance.

Fig. $8 \mathrm{~b}$ shows the equivalent magnetic noise spectral densities associated to the Fig. 8a after dividing by the sensitivity of the measuring chain. Although the sensor, with the new pick-up coil, reaches an equivalent magnetic white noise level below $0.6 \mathrm{pT} / \sqrt{\mathrm{Hz}}$ at frequencies higher than $300 \mathrm{~Hz}$ (gray triangles in Fig. 8b), the performances at very low frequencies are worse than those with the original pick-up coil (green squares in Fig. 8b). Strong correlation (between the generator and sensor noise spectral densities with new coil in its best performance) shows that, in this particular case, the generator noise is responsible for the noise behavior in the low frequency range (below $300 \mathrm{~Hz}$ ).

\section{Conclusion}

Off-diagonal magnetoimpedance sensor employing amorphous ribbon with creep-induced anisotropy was examined in order to investigate its magnetic noise performances. Based on detailed experimental procedures, a successful approach to sub-pT resolution was achieved with an improved operation set-up of the sensor. A reduction of white noise level from about $5 \mathrm{pT} / \sqrt{\mathrm{Hz}}$ [7] to $560 \mathrm{fT} / \sqrt{\mathrm{Hz}}$ has been achieved for frequencies higher than $300 \mathrm{~Hz}$. Our experiments also proved the fact that resolution of magnetoimpedance sensors is mainly limited by electronic devices that operate the sensors. Hence, the intrinsic magnetic noise level of magnetoimpedance sensor is difficult to observe without deep characterization of full measurement of the operation set-up.

The experiments also point out the irreversible transformation of the magnetic structure in the ribbons occurring with high amplitude excitation. At certain amplitude of AC current, the magnetic ordering in the ribbon changes from circumferentially oriented magnetization moments into a domain structure with left- and right-hand oriented magnetic domains and the $1 / f$ noise component of the sensor dramatically increases which may be a consequence of domain walls (domain wall movements) presence in the structure. The point, at which the magnetic structure changes, depends on the excitation current amplitude, on the DC bias current and also on the external static magnetic field working point. Further work on this area has to be done to improve the low frequency equivalent magnetic noise of GMI-based sensor.

\section{Acknowledgments}

Authors would like to thank to LUDĚK KRAUS for his valuable remarks and for preparation of the ferromagnetic material that was used in the sensor's core.

[1] M. Knobel, M. Vazquez, L. Kraus, Giant magnetoimpedance, Handbook of magnetic materials 15 (2003) 497-563.

[2] I. Sasada, Orthogonal fluxgate mechanism operated with dc biased excitation, Journal of Applied Physics 91 (10) (2002) 7789-7791.

[3] B. Dufay, S. Saez, C. Dolabdjian, D. Seddaoui, A. Yelon, D. Ménard, Improved gmi sensors using strongly-coupled thin pick-up coils, Sensor Letters 7 (3) (2009) 334-338.

[4] M. Malatek, Ph.D. thesis, CTU in Prague (2007).

[5] L. Kraus, M. Malátek, M. Dvořák, Magnetic field sensor based on asymmetric inverse wiedemann effect, Sensors and Actuators A: Physical 142 (2) (2008) 468-473.

[6] A. Plotkin, E. Paperno, A. Samohin, I. Sasada, Compensation of the thermal drift in the sensitivity of fundamental-mode orthogonal fluxgates, Journal of applied physics 99 (8) (2006) 08B305-08B305.

[7] M. Malátek, L. Kraus, Off-diagonal gmi sensor with stress-annealed amorphous ribbon, Sensors and Actuators A: Physical 164 (1) (2010) 41-45.

[8] B. Dufay, S. Saez, C. Dolabdjian, A. Yelon, D. Menard, Impact of electronic conditioning on the noise performance of a two-port network giant magnetoimpedance magnetometer, Sensors Journal, IEEE 11 (6) (2011) 1317-1324.

[9] L. Ding, S. Saez, C. Dolabdjian, L. Melo, A. Yelon, D. Menard, Equivalent magnetic noise limit of low-cost gmi magnetometer, Sensors Journal, IEEE 9 (2) (2009) 159-168.

[10] S. Yabukami, H. Mawatari, N. Horikoshi, Y. Murayama, T. Ozawa, K. Ishiyama, K. Arai, A design of highly sensitive gmi sensor, Journal of magnetism and magnetic materials 290 (2005) 1318-1321.

[11] L. Melo, D. Menard, A. Yelon, L. Ding, S. Saez, C. Dolabdjian, Optimization of the magnetic noise and sensitivity of giant magnetoimpedance sensors, Journal of Applied Physics 103 (3) (2008) 033903-033903.

[12] B. Dufay, S. Saez, C. Dolabdjian, A. Yelon, D. Menard, Characterization of an optimized off-diagonal gmibased magnetometer, Sensors Journal, IEEE 13 (1) (2013) 379-388.

[13] B. Dufay, S. Saez, C. Dolabdjian, A. Yelon, D. Menard, Development of a high sensitivity giant magnetoimpedance magnetometer: Comparison with a commercial flux-gate, Magnetics, IEEE Transactions on 49 (1) (2013) 85-88.

[14] M. Butta, S. Yamashita, I. Sasada, Reduction of noise in fundamental mode orthogonal fluxgates by optimization of excitation current, Magnetics, IEEE Transactions on 47 (10) (2011) 3748-3751. 


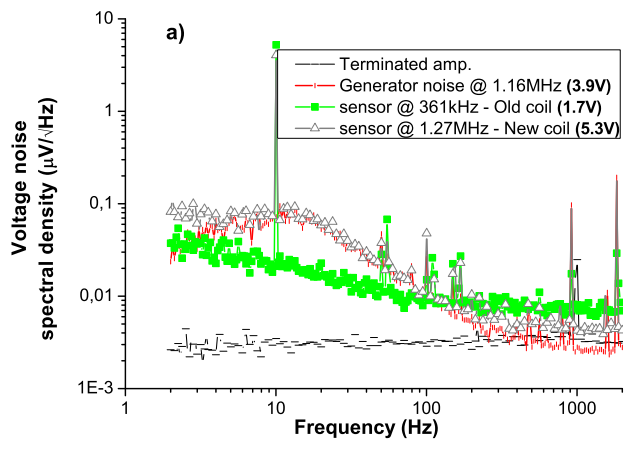

(a)

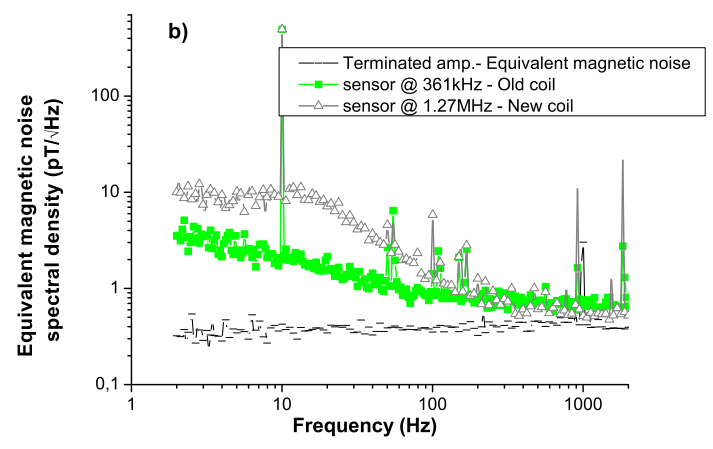

(b)

Figure 8: Voltage (a) and equivalent magnetic (b) noise spectral density versus experimental condition. The best performances achieved with the original coil and the one with the new coil is given by the green full squares and empty gray triangles, respectively. White noise floor is limited by the detection stage noise level (minus black marks). The noise level of the generator dynamic range is given by the red line. 